\title{
Relação entre condição de estresse emocional e presença de inflamação gengival em mulheres
}

Relationship between emotional stress condition and presence of gingival inflammation in women Relación entre la condición de estrés emocional y la presencia de inflamación gingival en mujeres Júlia Tavares PALMEIRA 1 Itamar da Silva NUNES 2 Waleska Fernanda SOUTO-NÓBREGA João Nilton Lopes de SOUSA ${ }^{4}$

'Graduanda em Odontologia, Universidade Federal de Campina Grande - UFCG, 58428-830 Patos - PB, Brasil ${ }^{2}$ Cirurgião-Dentista, Universidade Federal de Campina Grande - UFCG, 58428-830 Patos - PB, Brasil ${ }^{3}$ Doutoranda em Clínicas Odontológicas, PPGO/ Universidade Estadual da Paraíba - UEPB, 58429-500 Campina Grande - PB, Brasil ${ }^{4}$ Professor Doutor do curso de Odontologia, Universidade Federal de Campina Grande- UFCG, 58428-830 Patos - PB, Brasil

\section{Resumo}

Condições sistêmicas do hospedeiro, entre estas, os hormônios sexuais, tem sido apontadas como fator relevante que pode influenciar a patogênese das doenças periodontais. O presente trabalho objetivou avaliar o índice de prevalência de inflamação gengival em mulheres a partir de 30 anos em condição de estresse emocional atendidas na clínica escola de Odontologia da UFCG. Ao fim, foram incluídas no estudo o equivalente a 115 fichas de mulheres atendidas na disciplina de Periodontia em clínica multidisciplinar entre Maio de 2012 e Fevereiro de 2014. Foi realizada análise multivariada através da Correlação de Pearson através do software SPSS versão 22.0. Foi verificado que $90 \%$ da amostra possuía características de inflamação gengival. O resultado da equação apresentou valor igual a um, indicando que há uma correlação linear positiva perfeita - maior valor que pode ser apresentado entre o estresse e a inflamação gengival.

Descritores: Estresse; Mulher; Periodontia.

\section{Abstract}

Host systemic conditions, including sex hormones, have been identified as a relevant factor that may influence the pathogenesis of periodontal diseases. The present study aimed to evaluate the prevalence rate of gingival inflammation in women aged 30 years and under emotional stress attended at the UFCG School of Dentistry. In the end, the study included the equivalent of 115 records of women treated in the Periodontics course in multidisciplinary clinic between May 2012 and February 2014. Multivariate analysis was performed using Pearson Correlation using SPSS version 22.0 software. It was found that $90 \%$ of the sample had gingival inflammation characteristics. The result of the equation presented a value of one, indicating that there is a perfect positive linear correlation - the highest value that can be presented between stress and gingival inflammation.

Descriptors: Stress; Woman; Periodontics.

\section{Resumen}

Las condiciones sistémicas del huésped, incluidas las hormonas sexuales, se han identificado como un factor relevante que puede influir en la patogénesis de las enfermedades periodontales. El presente estudio tuvo como objetivo evaluar la tasa de prevalencia de inflamación gingival en mujeres de 30 años y bajo estrés emocional atendidas en la Facultad de Odontología de UFCG. Al final, el estudio incluyó el equivalente a 115 registros de mujeres tratadas en el curso de Periodoncia en una clínica multidisciplinaria entre mayo de 2012 y febrero de 2014. El análisis multivariado se realizó con Pearson Correlation con el software SPSS versión 22.0. Se encontró que el 90\% de la muestra tenía características de inflamación gingival. El resultado de la ecuación presentó un valor de uno, lo que indica que existe una correlación lineal positiva perfecta, el valor más alto que se puede presentar entre el estrés y la inflamación gingival.

Descriptores: Estrés; Mujer; Periodoncia.

INTRODUÇÃO

Dentre as diversas patologias que acometem o ambiente bucal, a doença periodontal se destaca por sua alta prevalência. Esta doença caracteriza-se como uma doença infecciosa inflamatória, que acomete tanto os tecidos de proteção como os tecidos de sustentação do periodonto. Sabe-se, na atualidade, que a presença de biofilme dentário, constituído por depósitos bacterianos e constituintes salivares, está diretamente ligada a instalação e progressão da doença periodontal, tornando-a uma doença biofilme dependente ${ }^{1}$.

Sendo assim, os fatores / condições sistêmicas do hospedeiro devem ser consideradas. Entre estes, os hormônios sexuais têm sido apontados como um fator relevante que podem influenciar a patogênese das doenças periodontais ${ }^{2}$. Os hormônios são moléculas reguladoras específicas que têm efeitos potentes sobre os principais determinantes do desenvolvimento e a integridade do esqueleto e da cavidade bucal, incluindo os tecidos periodontais. Está claro que as manifestações periodontais ocorrem quando há 0 desequilíbrio desses hormônios esteroides ${ }^{3}$.

Em condições onde a influência hormonal predispõe ao desenvolvimento de doenças periodontais ou exacerba processos inflamatórios periodontais preexistentes, o tratamento periodontal deve ser capaz de eliminar a resposta inflamatória, onde ao mesmo tempo devem ser realizados o controle e manutenção do tratamento, bem como a orientação dos pacientes acerca dos cuidados essenciais com sua higiene oral para que estes tenham a autonomia necessária para realizarem a escovação e o uso de fio dental corretamente. Pode-se perceber que o emprego da terapêutica periodontal influenciar na obtenção de uma melhor qualidade de vida para as mulheres que se encontram em fases de estresse da vida, onde há a possibilidade de ocorrer interferência hormonal nos problemas periodontais ${ }^{4}$.

Em seu estudo Domingos et al. ${ }^{5}$ abordaram diferentes estratégias metodológicas 
para investigar a influência do estresse psicológico na periodontite e concluíram que pode haver alguma relação entre os fatores psicológicos negativos e a manifestação ou progressão da periodontite, sugerindo que $s$ realizem mais estudos para esclarecer através de quais mecanismos os fatores psicológicos causam efeitos sobre a periodontite ainda não estão claros. Também é possível relacionar o estresse psicológico com a Periodontia no fato de que este é um dos fatores da etiopatogenia da doença periodontal através da interação com - sistema neuroendócrino imunológico. Entretanto ainda há limitado número de estudos com metodologia confiável e falta de concordância de resultados para confirmação dessa informação ${ }^{6}$.

Pensando-se na família como um espaço primário de relacionamento social, as mulheres e mães exercem uma influência especial, principalmente nas questões relacionadas à saúde, pois atuam como agentes produtoras e multiplicadoras de conhecimentos, informações e atitudes que visam à promoção da sua saúde e a de toda a sua família ${ }^{7}$.

Destarte, o presente trabalho objetivou avaliar o índice de prevalência de inflamação gengival em mulheres a partir de 30 anos em condição de estresse emocional na clínica escola da UFCG.

\section{MATERIAL E MÉTODO}

Trata-se de um estudo transversal, quantitativo de caráter exploratório que objetivou avaliar o índice de prevalência de inflamação gengival em mulheres a partir de 30 anos em condição de estresse emocional atendidas na clínica escola de Odontologia da UFCG. Ao fim, foram incluídas no estudo o equivalente a 115 fichas de mulheres atendidas na disciplina de Periodontia em clínica multidisciplinar entre Maio de 2012 e Fevereiro de 2014. Foi realizada análise multivariada através da Correlação de Pearson utilizando software SPSS versão 22.0. O coeficiente de correlação de Pearson ( $r$ ) ou coeficiente de correlação produto-momento ou o $r$ de Pearson mede o grau da correlação linear entre duas variáveis quantitativas, de forma adimensional com valores situados ente -1,0 e 1.0 inclusive, refletindo a intensidade de uma relação linear entre dois conjuntos de dados. Se $R=1$ significa uma correlação perfeita positiva entre as duas variáveis; se $R=-1$ significa uma correlação negativa perfeita entre as duas variáveis, Logo, se uma aumenta, a outra sempre diminui e $R=0$ significa que as duas variáveis não dependem linearmente uma da outra ${ }^{8}$.
RESULTADOS

A Tabela 1 mostra o grau de instrução em percentuais de mulheres que participaram da pesquisa, comparando com o valor total da amostra. De acordo com os dados abaixo, o maior valor encontrado foi Ensino Médio Completo com 70,9\%.

Tabela 1. Grau de escolaridade e sexo dos pacientes, conforme amostra utilizada, da Escola de Odontologia da Universidade Federal de Campina Grande - Campus Patos, 2019

\begin{tabular}{c|c|c|c}
\hline Grau de Instrução & Mulheres & $\begin{array}{c}\text { Total da } \\
\text { amostra }\end{array}$ & Mulheres (\%) \\
\hline Analfabetismo & $\mathbf{2}$ & 7 & $28,6 \%$ \\
\hline Ens. Fund. Incompleto & 25 & 35 & $71,4 \%$ \\
\hline Ens. Fund. Completo & 13 & 16 & $81,3 \%$ \\
\hline Ens. Médio Incompleto & 9 & 17 & $52,9 \%$ \\
\hline Ens. Médio Completo & 39 & 55 & $70,9 \%$ \\
\hline Ens. Superior Incompleto & 17 & 31 & $54,8 \%$ \\
\hline Ens. Superior Completo & 10 & $\mathbf{1 4}$ & $71,4 \%$ \\
\hline TOTAL & $\mathbf{1 1 5}$ & $\mathbf{1 7 5}$ & $\mathbf{6 5 , 7 \%}$ \\
\hline
\end{tabular}

Segundo a população estudada, é possível constatar que mulheres de trinta a sessenta anos, correspondem o maior valor, entretanto é possível analisar que mulheres abaixo de quinze anos, não buscam e nem são direcionadas para atendimentos em periodontia correspondendo $0,9 \%$ da amostra total (Tabela 2).

Tabela 2. Faixa etária (em anos) das pacientes da Escola de Odontologia, consoante amostra utilizada, da Universidade Federal de Campina Grande - Campus Patos, 2019.

\begin{tabular}{l|c|c}
\hline Faixa etária & Quantidade & (\%) \\
\hline Abaixo de 15 anos & 1 & $0,9 \%$ \\
\hline 20 |----- 30 & 44 & $38,3 \%$ \\
\hline 30 ----- 60 & 66 & $57,4 \%$ \\
\hline Acima de 60 anos TOTAL & $\mathbf{1 1 5}$ & $3,5 \%$ \\
\hline \multicolumn{2}{|c|}{$\mathbf{1 0 0 , 0 \%}$} \\
\hline Fonte: Dados da pesquisa
\end{tabular}

De acordo com a Tabela 3, é possível deduzir que os maiores índices de fatores predisponentes locais, são cálculo dentário, diastema e recessão gengival com níveis percentuais comparativos com o universo da amostra de $62,1 \%$, 66,2\% e $56,0 \%$ respectivamente.

As repercussões das complicações sistêmicas também podem repercutir em cavidade bucal e os dados apresentados na Tabela 3, demonstram que esses fatores são bastante relevantes. O uso do anticoncepcional, hábito de beber, paciente fumante ou extabagista e o estresse foram os maiores dados obtidos, sendo o estresse com o maior índice.

De todos os dados coletados, a quantidade de mulheres que estão em condição de gengiva inflamada corresponde a um número de grande relevância. Em todos os dados da amostra do sexo feminino, $90 \%$ da amostra possui a gengiva inflamada e apenas 10\% possui a gengiva saudável (Gráfico 1).

Com um número bastante expressivo, a condição do estresse está presente em muitos 
pacientes atualmente e necessita de uma abordagem maior. A tabela abaixo mostra a correlação de Pearson utilizada para avaliar até que ponto as idades - de mulheres a partir de 30 anos - influenciam no desenvolvimento condições de estresse e inflamação gengival mediante as quantidades coletadas.

Tabela 3. Fatores predisponentes locais e sistêmicos apresentados pelos pacientes da Escola de Odontologia da Universidade Federal de Campina Grande - Campus Patos, 2019

\begin{tabular}{|c|c|c|c|}
\hline $\begin{array}{c}\text { Fatores Predisponentes } \\
\text { Locais }\end{array}$ & Mulheres & $\begin{array}{l}\text { Total da } \\
\text { amostra }\end{array}$ & Mulheres (\%) \\
\hline Ausentes & 17 & 23 & $73,9 \%$ \\
\hline Presentes & 98 & 152 & $64,4 \%$ \\
\hline Cálculo Dentário & 36 & 58 & $62,1 \%$ \\
\hline Diastema & 49 & 74 & $66,2 \%$ \\
\hline Giroversão & 41 & 63 & $65,1 \%$ \\
\hline Apinhamento & 28 & 42 & $66,7 \%$ \\
\hline Recessão Gengival & 47 & 84 & $56,0 \%$ \\
\hline Alteração Oclusal & 22 & 32 & $68,8 \%$ \\
\hline Aparelho Ortodôntico & 7 & 10 & $70,0 \%$ \\
\hline $\begin{array}{c}\text { Posição Inadequada de } \\
\text { Freios e Bridas }\end{array}$ & 13 & 20 & $65,0 \%$ \\
\hline Fatores Sistêmicos & Mulheres & $\begin{array}{c}\text { Total da } \\
\text { amostra } \\
\text { (homens e } \\
\text { mulheres) }\end{array}$ & Mulheres (\%) \\
\hline Ausentes & 31 & 70 & $44,3 \%$ \\
\hline Presentes & 84 & 105 & $80,0 \%$ \\
\hline Diabetes & 1 & 3 & $33,3 \%$ \\
\hline Cardiopatia & 4 & 7 & $57,1 \%$ \\
\hline Hipertensão & 7 & 9 & $77,8 \%$ \\
\hline Estresse & 48 & 61 & $78,7 \%$ \\
\hline Etilismo & 25 & 46 & $54,3 \%$ \\
\hline Fumante ou ex-fumante & 20 & 27 & $74,1 \%$ \\
\hline Anticoncepcional & 20 & 20 & $100,0 \%$ \\
\hline
\end{tabular}

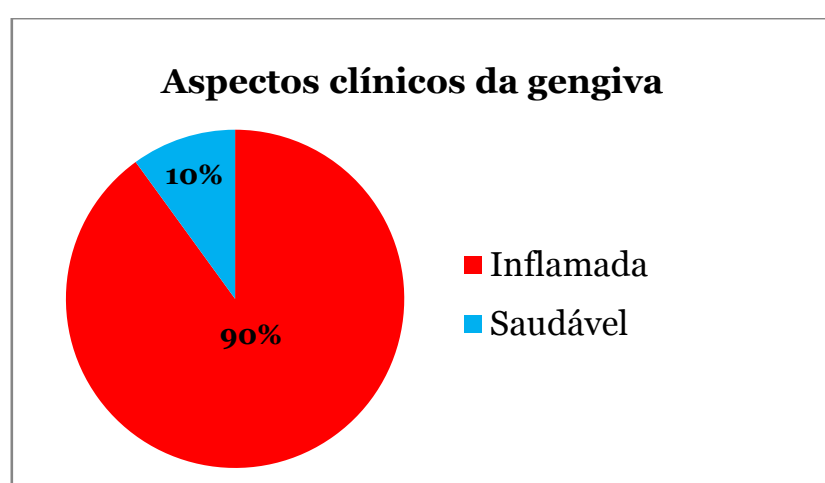

Gráfico 1: Nível de inflamação gengival em mulheres mediante ao levantamento de dados da Escola de Odontologia da Universidade Federal de Campina Grande - Campus Patos, 2019 (Fonte: Dados da pesquisa).

Os dados distribuídos na Tabela 4 representam as variáveis ( $x$ e $y$ ) analisadas $e$ indicadas pela quantidade de mulheres a partir de trinta anos de idade (de trinta a sessenta e acima de sessenta) como termo independente valores de $\mathrm{x}$ - e a quantidade tanto dos fatores de inflamação quanto os de estresse como termos dependentes - valores de y. Isso significa que, será buscado no cálculo identificar se há algum tipo de dependência da inflamação e estresse em mulheres com idades acima de trinta anos (fator independente). O resultado da equação apresenta valor igual a um, ou seja, isso indica que há uma correlação linear positiva perfeita - maior valor que pode ser apresentado. Nesse sentido, é possível inferir que há uma forte associação estabelecida a partir do quantitativo das idades das mulheres acima de trinta anos com condições de estresse e inflamação gengival.

Tabela 4. Cálculo da correlação entre as idades a partir de trinta anos e os fatores de inflamação e estresse apresentados pelas pacientes na Escola de Odontologia da Universidade Federal de Campina Grande - Campus Patos, 2019

\begin{tabular}{|c|c|c|c|c|c|c|}
\hline Idades (x) & Quantidade & $\begin{array}{c}\text { Fatores } \\
\text { analisados (y) }\end{array}$ & Quantidade & $\mathbf{X}^{2}$ & $\mathbf{y}^{2}$ & $\mathbf{x} \cdot \mathbf{y}$ \\
\hline 30|----- 60 & 66 & Inflamação & 90 & 4356 & 8100 & 5940 \\
\hline $\begin{array}{l}\text { Acima de } 60 \\
\text { anos }\end{array}$ & 4 & Estresse & 48 & 16 & 2304 & 192 \\
\hline Total & 70 & - & 138 & 4372 & 10404 & 6132 \\
\hline
\end{tabular}

DISCUSSÃO

Sabe-se que a doença periodontal acontece apenas quando ocorre um desequilíbrio entre a resposta do hospedeiro e a agressão causada pelos microrganismos. Assim, sempre que se estuda se determinado hábito ou alteração sistêmica seja ela congênita ou adquirida, deve ser visto como um fator de risco para a doença periodontal deve-se considerar $o$ que isto pode acarretar na resposta/ resistência do hospedeiro.

Os mecanismos do estresse e doença periodontal são ainda, em muitos pontos, de difícil compreensão. Sabe-se que o estresse conduz a mudanças no eixo hipotálamopituitário-adrenal (HPA), capaz de interferir nas intercomunicações dos sistemas, suprimindo o sistema de defesa, estabelecendo-se a doença. $\mathrm{Na}$ doença periodontal existem estudos em animais e humanos, que ligam o estresse e suas vias à doença periodontal ${ }^{9}$.

A incidência de gengivite ulcerativa necrosante aumenta durante períodos de estresse emocional e fisiológico, sugerindo uma ligação entre os dois. O estresse emocional pode interferir com a função imune normal, podendo resultar em aumento dos níveis de hormônio circulantes, que podem ter impacto no periodonto $^{10}$.

Os principais hormônios sexuais, que exercem influência sobre o periodonto, são estrógeno e progesterona ${ }^{11,12}$. Parece haver uma associação direta entre aumento dos níveis destes hormônios e aumento do número de microrganismos anaeróbicos, que possuem um papel importante no desencadeamento e progressão da doença periodontal, principalmente diminuindo a capacidade fagocítica dos leucócitos polimorfonucleares (PMN) e aumentando a secreção de interleucina 1b (IL-1b). Além disso, os hormônios sexuais aumentam a permeabilidade vascular e também 
incrementam a interação de enzimas proteolíticas com a interleucina 6 (IL-6), um mediador da inflamação ${ }^{13}$.

Em um estudo que sugere uma associação entre depressão crônica e condição periodontal em mulheres, foi observado que o grupo com depressão crônica possuía periodontite mais severa e altas concentrações de interleucina 6 (IL-6) no fluido gengival que mulheres consideradas saudáveis. As alterações imunológicas nestas pacientes são consideradas um reflexo da exposição crônica ao estresse e podem contribuir para a piora das condições periodontais ${ }^{14}$.

O padrão da gengivite parece seguir 0 ciclo normal das alterações hormonais e pode sofrer uma variação significativa de estadiamento. Uma mudança de microbiota, propiciada por estas alterações hormonais, também é notada, principalmente o aumento expressivo de Prevotella intermedia e Porphyromonas gingivalis ${ }^{15}$.

$\mathrm{O}$ estresse emocional pode alterar a secreção de produtos de defesa do hospedeiro, como por exemplo, diminuindo a produção de imunoglobulina-A $(\lg A)$. A $\lg A$ é o anticorpo predominante na saliva e pode ser considerado um dos agentes antibacterianos mais importantes para a manutenção da homeostase. A sua diminuição provoca destruição tecidual ativada por produtos bacterianos, provavelmente, mediada por meio de citocinas, liberadas por células do sistema imune ativadas, entre outras, levando a um desequilíbrio na relação parasita-hospedeiro ${ }^{16}$.

No organismo feminino essas mudanças a nível hormonal transcorrem nas várias fases da vida. Nesse contexto, a homeostase dos tecidos periodontais mostra-se prejudicada, no entanto, a inflamação gengival não ocorre somente em decorrência da ação hormonal. Para que se instale o processo inflamatório atuam outros fatores desencadeadores, tais como: biofilme bacteriano com bactérias específicas predisponentes a doença periodontal e resistência imunológica do hospedeiro ${ }^{17-19}$.

A importância do estresse como um possível fator de risco para a doença periodontal é indiscutível ${ }^{20}$. Os trabalhos publicados até o momento, em sua grande maioria, têm mostrado que quadros de estresse que acometem indivíduos com pobres estratégias para o seu manuseio podem alterar significativamente a resposta do hospedeiro, tanto pela via imunológica, como pela via comportamental.
CONCLUSÃO

Diante do exposto, a correlação de estresse em mulheres exerce impacto expressivo na inflamação gengival, embora não sejam necessários ou suficientemente capazes de desencadear, por si só, mudanças gengivais. Contudo, estas podem alterar as respostas periodontais frente ao insulto bacteriano, o que, indiretamente, contribuiria com a doença periodontal.

REFERÊNCIAS

1. Santos JF, Pillon FL. A influência dos hormônios sexuais femininos sobre a manifestação clínica das doenças periodontais: revisão de literatura. Periodontia. 2009; 19(3):34-40.

2. Mascarenhas $P$, Gapski R, Al-Shammari K, Wang HL. Influence of sex hormones on the periodontium. J Clin Periodontol. 2003;30(8):671-81.

3. Güncü GN, Tözüm TF, Cağlayan F. Effects of endogenous sex hormones on the periodontium--review of literature. Aust Dent $\mathrm{J}$. 2005;50(3):138-45

4. Spezzia S. Interrelationship between Sex Hormones and Periodontal Diseases in Women. Periodontia. 2016;26(2):40-7.

5. Domingos AL. A influência do estresse psicológico na doença periodontal: uma revisão de literatura [monografia]. Florianópolis: Universidade Federal de Santa Catarina (UFSC); 2016.

6. Balderrama IF, et al. Are salivary markers sufficient to correlate psychological stress and periodontal disease? Narrative review of literature. Periodontia. 2017;27(2):67-74.

7. Costa ICC. Atenção odontológica à gestante na triangulação médico- dentista-paciente [tese]. Araçatuba: Programa de Pós-Graduação em Odontologia Preventiva e Social (UNESP); 2000.

8. Figueiredo Filho DB, Silva Júnior JA. Desvendando os Mistérios do Coeficiente de Correlação de Pearson (r) ${ }^{*}$. Rev Política Hoje. 2009;18(1):115-46.

9. Segundo AS, Semenoff TDV, Bosco AF, Biazoli ER, Ribeiro RV, Rocatto GEGD et al. Efeito do estresse crônico na progressão da periodontite induzida por ligadura em ratos. $\mathrm{R}$ Periodontia. 2007;17(3):62-6.

10. Newman MG, et al. Periodontia Clínica. 10. ed. Rio de Janeiro: Elsevier; 2007.

11. Jönsson $D$. The biological role of the female sex hormone estrogen in the periodontium-studies on human periodontal ligament cells. Swed Dent J Suppl. 2007;(187):11-54.

12. Markou E, Eleana B, Lazaros T, Antonios K. The influence of sex steroid hormones on gingiva of women. Open Dent J. 2009;3:114-9. 
13. Koreeda $\mathrm{N}$, Iwano $\mathrm{Y}$, Kishida $\mathrm{M}$, Otsuka $\mathrm{A}$, Kawamoto A, Sugano N, Ito K. Periodic exacerbation of gingival inflammation during the menstrual cycle. J Oral Sci. 2005;47(3):159-64.

14. Johannsen A, Rydmark I, Söder B, Asberg M. Gingival inflammation, increased periodontal pocket depth and elevated interleukin- 6 in gingival crevicular fluid of depressed women on long-term sick leave. J Periodontal Res. 2007;42(6):546-52.

15. Krejci CB, Bissada NF. Women's health issues and their relationship to periodontitis. J Am Dent Assoc. 2002;133(3):323-29.

16. Ayub LG, Novaes Júnior $A B$, Grisi MFM, Souza SLS, Palioto DB, LeitePanassi CRA et al. Estresse como possível fator de risco para a doença periodontal - revisão de literatura. $R$. Periodontia. 2010;20(3):28-36.

17. Hugoson A. Gingival inflammation and female sex hormones. A clinical investigation of pregnant women and experimental studies in dogs. J Periodontal Res Suppl. 1970;5:1-18.

18. Axelsson $\mathrm{P}$, Lindhe $\mathrm{J}$, Wäseby J. The effect of various plaque control measures on gingivitis and caries in schoolchildren. Community Dent Oral Epidemiol. 1976;4(6):232-39 .

19. de Liefde $B$. The dental care of pregnant women. N Z Dent J. 1984 Apr;80(360):41-3.

20. Justo MP, Jardim Junior EG, Schweitzer CM. Estresse, depressão e imunidade a infecções. Arch Health Invest 2017;6(Spec Iss 4):42.

\section{CONFLITO DE INTERESSES}

Os autores declaram não haver conflitos de interesse

AUTOR PARA CORRESPONDÊNCIA

Waleska Fernanda Souto Nóbrega

Rua Francisca Edith - 155, apto. 203, Centro, 58600000 Santa Luzia - PB, Brasil

Fone: (83)99631 2031

Email: drawaleskasouto@gmail.com 